\title{
Steady MHD Flow of Viscous Fluid between Two Parallel Porous Plates with Heat Transfer in an Inclined Magnetic Field
}

\author{
D. R. Kuiry ${ }^{1}$, S. Bahadur ${ }^{2 *}$ \\ ${ }^{1}$ P.G. Department of Mathematics, Kolhan University, Chaibasa-833202, Jharkhand, India \\ ${ }^{2}$ Department of Mathematics, R.V.S. College of Engineering and Technology, \\ Jamshedpur-831012, India
}

Received 14 March 2015, accepted in final revised form 22 April 2015

\begin{abstract}
The steady flow behavior of a viscous, incompressible and electrically conducting fluid between two parallel infinite insulated horizontal porous plates with heat transfer is investigated along with the effect of an external uniform transverse magnetic field, the action of inflow normal to the plates, the pressure gradient on the flow and temperature. The fluid viscosity is supposed to vary exponentially with the temperature. A numerical solution for the governing equations for both the momentum transfer and energy transfer has been developed using the finite difference method. The velocity and temperature distribution graphs have been presented under the influence of different values of magnetic inclination, fluid pressure gradient, inflow acting perpendicularly on the plates, temperature dependent viscosity and the Hartmann number. In our study viscosity is shown to affect the velocity graph. The flow parameters such as viscosity, pressure and injection of fluid normal to the plate can cause reverse flow. For highly viscous fluid, reverse flow is observed. The effect of magnetic force helps to restrain this reverse flow.
\end{abstract}

Keywords: Heat transfer; Temperature dependent; Porous plates; Conducting fluid; Magnetic field.

(c) 2015 JSR Publications. ISSN: 2070-0237 (Print); 2070-0245 (Online). All rights reserved. doi: http://dx.doi.org/10.3329/jsr.v7i3.22574 J. Sci. Res. 7 (3), 21-31 (2015)

\section{Introduction}

The magnetohydrodynamic fluid flow between two parallel plates has wide applications in various fields such as petroleum industries, power generators, polymer technology, aerodynamic heating and also in the purification of crude oil. Steady viscous fluid flow between two plates under the action of magnetic force is utilized in bearings with liquidmetal lubricators. Channel-flow problems are practically important when the effect of

* Corresponding author: suryabahadur550@gmail.com 
viscosity and its variation is included. It has wide applications in lubrication based machinery.

Magnetohydrodynamic (MHD) is the science of motion of an electrically conducting fluid like mercury, molten iron and ionized gases known as plasma under the influence of magnetic field. Other names such as hydromagnetics, magnetic fluid mechanics are also in use. When a conductor moves in a magnetic field, the two consequences arise. Firstly, the electric current is induced and this current experiences a force known as Lorentz force and secondly an induced magnetic field is produced and is added to the primitive magnetic field [1-4]. Such type of study is mostly coined, concentrated and enunciated extensively by Alfvén [5] in 1942. A classical problem accorded with the hydromagnetic channel flow yields the exact solutions [6].

The exact solutions for the fluid and temperature fields of simplified problems can be found through the references [7-9]. The laminar flow governing equations in a channel are generally the Navier-Stoke's and energy equations and theses equations are mutually coupled and reduce to non-linear form when there is a temperature dependent fluid viscosity and consequently it results in the effect of viscosity variation or viscous dissipation [10-13]. A few property laws can help to solve the hydrodynamic problem to account for variable viscosity and to avoid the coupling of the governing equations.

In the present note, the steady flow behavior of an electrically conducting, viscous, incompressible fluid bounded by two parallel infinite insulated horizontal porous plates under the action of an external uniform magnetic field applied perpendicularly to the plates is studied with the assumption of the fluid viscosity to vary exponentially with the temperature and the plates are kept at different but constant temperatures. The fluid motion is also under the influence of a uniform suction and injection at the upper and lower plates respectively. The upper plate moves with a horizontal uniform velocity and is subjected to a pressure gradient in the horizontal direction. The fluid flow and the temperature distributions are governed by the coupled set of the mass conservation equation, momentum transfer equation and the energy equation and consequently the nonlinear ordinary differential equations are solved numerically employing the finite difference methods. The effects of the inclined magnetic field, variable viscosity on both the flow and temperature at various inclinations of the magnetic field and Hartmann number are studied through graphical representations.

\section{Mathematical formulation of the problem}

Let the viscous, incompressible and electrically conducting fluid move with velocity $\vec{V}$ between two parallel infinite insulated horizontal porous plates under the influence of a magnetic field $\overrightarrow{B_{0}}$ acted perpendicularly upon the plates. The origin is located in the lower plate with $\mathrm{x}$-axis along the main flow and $\mathrm{y}$-axis perpendicular to both the plates. The plates are assumed to be infinitely long in the $\mathrm{x}$ and $\mathrm{z}$-directions. The layer in contact with the lower plate is obviously on XOZ plane. Consequently the problem becomes onedimensional and the fluid velocity component $u$ in the x-direction and the temperature $T$, 
both are functions of $\mathrm{y}$ only except the pressure gradient term in the direction of $\mathrm{x}$-axis. Due to no slip condition at the lower plate with no motion, the component $\mathrm{u}$ is zero and using the mass conservation equation the y-component of the fluid velocity $v$ is always constant and is equal to the suction velocity $v_{s}$ whereas at the upper plate the component $u$ is equal to the velocity of the plate $u_{1}$. The uniform magnetic field $\overrightarrow{B_{0}}$ is directed along the direction of y-axis by neglecting the induced electric and magnetic fields. The electromagnetic influence is employed to restrain the fluid motion subject to imposition of a force proportional to the fluid velocity which is under the effect of a pressure gradient in the x-direction. Also the fluid temperature $T$ at the lower plate is $T_{1}$ while that at the upper plateis $T_{2}$.

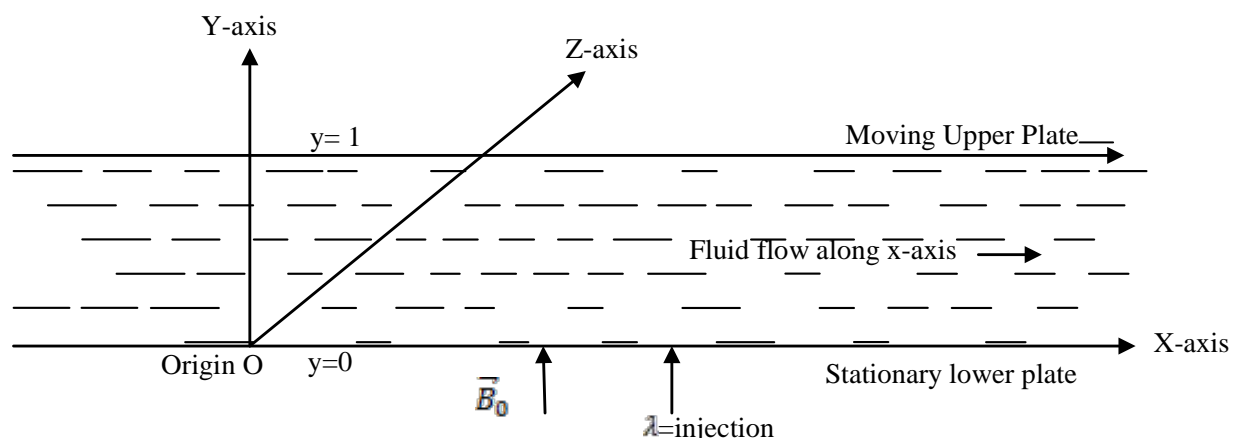

Fig. 1. Model of the problem.

The electric field vector $\vec{E}$ is produced [9] at right angles to both the fluid velocity $\vec{V}$ and the magnetic field $\vec{B}$ and is given by

$$
\vec{E}=\vec{V} \times \vec{B}
$$

Let the electrically conducting fluid be isotropic in spite of the magnetic field $\vec{B}$. As a result an electrical conductivity $\sigma$ will be related with electric current density $\vec{i}$ and electric field $\vec{E}$ given by Ohm's law [10] in the following manner;

$$
\vec{l}=\sigma(\vec{E}+\vec{V} \times \vec{B})
$$

where $\vec{V}=\left(u, v_{g}, 0\right)$ and $\vec{B}=\left(0, B_{0}, 0\right)$

or, $\quad \vec{J}=\sigma(\vec{V} \times \vec{B})$

The term $\sigma \vec{E}$ is negligible in comparison with the term $(\vec{V} \times \vec{B})$, if we take MHD approximations into account. 
Again at the same time the induced current denoted by the Lorentz force $\vec{F}$ is given by

$$
\vec{F}=\vec{J} \times \vec{B}
$$

which is being due to the electric generator, the conducting fluid and the temperature cut the lines of the magnetic field. The vector $\vec{F}$ is parallel to both the fluid velocity vector $\vec{V}$ and the temperature $T$ but in opposite directions. Such a laminar flow through parallel plates under the influence of the magnetic field has been studied by many authors in recent years [14-16] in the applications in MHD generator, MHD pumps and electromagnetic flow meters.

The momentum transfer and energy equations when applied to the fluid reduce to the forms $[17,18]$ given as below

$$
\rho v_{s} \frac{d u}{d y}=-\frac{d p}{d x}+\frac{d}{d y}\left(\mu \frac{d u}{d y}\right)-\sigma B_{0}^{2} u
$$

and

$$
\rho c v_{s} \frac{d T}{d y}=k \frac{d^{2} T}{d y^{2}}+\mu\left(\frac{d u}{d y}\right)^{2}+\sigma B_{0}^{2} u^{2}
$$

where $\rho=$ fluid density, $k=$ specific heat of the fluid, $\mu=$ fluid viscosity $=\mu_{0} \mu(T)$, $\mu_{0}=\rho v_{0}, v_{0}$ is the characteristic velocity of the fluid and $\mu_{0}$ is the characteristic viscosity of the fluid at $\mathrm{y}=0$.

Now, it is interesting to investigate the effect of an inclined magnetic field on the fluid velocity and the temperature distribution. This behavior is modeled by introducing an angle of inclination $\theta$ to the last terms of the right hand side of the equations (5) and (6) and these equations get modified respectively in the following manner:

$$
\rho v_{s} \frac{d u}{d y}=-\frac{d p}{d x}+\frac{d}{d y}\left(\mu \frac{d u}{d y}\right)-\sigma B_{0}^{2}\left(\sin ^{2} \theta\right) u
$$

and

$$
\rho c v_{s} \frac{d T}{d y}=k \frac{d^{2} T}{d y^{2}}+\mu\left(\frac{d u}{d y}\right)^{2}+\sigma B_{0}^{2}\left(\sin ^{2} \theta\right) u^{2}
$$

The equations (7) and (8) can be solved subject to the initial and boundary conditions:

$u=0, T=T_{1}$ at $y=0$

$u=u_{1}, T=T_{2}$ at $y=h$, where $\mathrm{h}$ is the distance between the two plates.

Substituting the following non-dimensional variables: 


$$
\begin{aligned}
& x^{*}=\frac{x}{h}, y^{*}=\frac{y}{h}, u^{*} \frac{w h}{v_{0}}, p^{*}=\frac{p h^{2}}{\rho v_{0}^{2}}, \\
& P^{*}=-\frac{d p^{*}}{d x^{*}}, P_{r}=\frac{\mu c}{k}, E_{c}=\frac{v_{0}^{2}}{h^{2} c\left(T_{2}-T_{1}\right)}, \lambda=\frac{h v_{s}}{v_{0}}
\end{aligned}
$$

$\lambda>0$ : injection, $\lambda<0$ : suction, $T^{*}=\frac{T-T_{1}}{T_{2}-T_{1}}$

$u_{1}^{*}=\frac{u_{1} h^{h}}{v_{0}}=R_{e}, R_{e}$ being the Reynolds number.

into the equations (7), (8) and (9) and dropping the stars for convenience, we get

$$
\begin{gathered}
\lambda \frac{d u}{d y}=P+\frac{d}{d y} \mu(T) \frac{d u}{d y}+\mu(T) \frac{d^{2} u}{d y^{2}}-M^{2} u \\
\lambda \frac{d T}{d y}=\frac{1}{P_{r}} \frac{d^{2} T}{d y^{2}}+E_{c} \mu(T)\left(\frac{d u}{d y}\right)^{2}+E_{c} M^{2} u^{2}
\end{gathered}
$$

Where $M=H_{\alpha} \sin \theta, H_{\alpha}=B_{0} h \sqrt{\frac{\sigma}{\mu}}, H_{\alpha}$ being the Hartmann number; $P_{\gamma} a n d E_{c}$ are the Prandtl and Eckert numbers respectively.

and

$u=0, T=0$ at $y=0$

$u=R_{e}, T=T_{1}$ at $y=1$

Equations (11) and (12) denote a system of coupled and non-linear ordinary differential equations subject to the conditions (13). Let the fluid viscosity vary exponentially with the temperature, then the function $\mu(T)$ takes the form (14) in the following manner:

$$
\mu(T)=e^{-\infty T}
$$

where $\alpha$ is the fluid viscosity parameter and is given by $\alpha=\log \left(\frac{\mu_{1}}{\mu_{2}}\right) ; \mu_{1}, \mu_{2}$ being the two values for the coefficients of fluid viscosity determined respectively at the lower plate temperature $T_{1}$ and at the upper plate temperature $T_{2}$.

\section{Numerical Solution of the Problem}

The equations (11) and (12) constitute a system of coupled and non-linear ordinary differential equations and can be solved subject to the initial and boundary conditions (13) employing a two- point finite difference methods [17,18].

Now let us introduce $\zeta$ and $\phi$ defined respectively by $\zeta=\frac{d u}{d y}$ and $\phi=\frac{d T}{d y}$. Using (14) along with these substitutions, the equations (11) and (12) reduce to the following forms respectively: 
and

$$
\zeta \lambda=P+e^{-\alpha T} \frac{d \zeta}{d y}-\alpha e^{-\omega T} \zeta \phi-M^{2} u
$$

$$
\lambda \phi=\frac{1}{P_{r}} \frac{d \phi}{d y}+E_{c} e^{-\omega T} \zeta^{2}+E_{c} M^{2} u^{2}
$$

subject to the initial and boundary conditions (13).

The governing non-linear ordinary differential equations can be solved through iteration techniques in which the computational domain has been divided into $N$ intervals in the y-direction and then in relation to the variables $\zeta$ and $\phi$. We obtain the finite difference equations by writing these equations at the mid-point of the computational cell and then again replacing the different terms by their second order central difference approximations. The difference equations obtained as such are to be solved numerically using Thomas' algorithm $[17,18]$.

\section{Results and Discussions}

The study of flow of an electrically conducting viscous fluid between two infinite horizontal plates requires rigorous calculations. Under various variable conditions i.e. the magnetic field with and without magnetic inclinations, fluid pressure gradient, inflow normal to the porous plates and temperature dependent viscosity we represent the velocity and temperature distributions through graphs. The non-dimensional velocity of the upper plate $u_{1}$ is taken to be unity which leads to a unit Reynolds number.

Fig. 2 represents the influence of pressure on the fluid flow in absence of $M$ and $\lambda$ but $\mu=1$. It shows the velocity distributions $u$ against the vertical distance $\mathrm{y}$ for various values of the pressure gradient $P$ from -3 to 3 . It is observed that for $P=-3$ i.e. for negative pressure gradient, reverse flow occurs, and for $P=-1$, the flow becomes linear. For $P=0$, the flow seems to be slightly above the line of linearity due to the presence of the fluid viscosity. When $P=3$, it is observed that the fluid velocity increases.

Fig. 3 shows that the effect of inflow due to injection on the velocity distribution for the values of $\lambda=0,2,6,10$ while $H a=3, \alpha=0, \mu=1, P=-3$, and $\theta=60^{\circ}$. It is seen that increasing $\lambda$ the reversed flow depth increases but when $\lambda=10$ taken arbitrarily, the recirculation flow depth reduces initially to some extent and suddenly the depth increases. Fig. 4 indicates the velocity profiles against the viscous parameter $\alpha$ in the presence of pressure. Setting the parameters $P=-5, \lambda=0, H a=0, T=1$. It is observed that the depth of the reversed flow increases with the increase of $\alpha$. It indicates that as viscosity increases, the reversed flow becomes deeper.

Fig. 5 reveals the effect of viscosity parameter $\alpha$ ranging from $-2,0,1,1.5$ on velocity profiles when the other parameters are taken as $M=0, P=0, \lambda=0, T=1$. In the absence of Hartmann number, pressure gradient, inflow, the viscosity induces substantial effect on the velocity distribution. As the viscosity parameter $\alpha$ increases, viscosity decreases and consequently velocity increases, it is observed that the mass flow rate indicated by the area between the flow velocity curves and the y-axis is reduced. It is also observed 
through the reference [4] that as $\alpha$ decrease, viscosity increases with temperature i.e. for the negative values of $\alpha$, the mass flow rate increases.

Fig. 6 shows the flow profiles for different values of Hartmann numbers (variable magnetic intensity), keeping the other parameters fixed. It is concluded that the depth of reversed flow reduces with an increase of magnetic field. For values of the viscosity parameter $\alpha$, it is observed that the increase of $\alpha$ reduces the mass flow rate which is indicated by the area between the flow velocity curves and the y-axis. It is also observed through the reference [4] that viscosity increases with temperature, in other words viscosity parameter $\alpha$ is of negative value, the mass flow rate increases as indicated in the Figure as $\alpha$ changes.

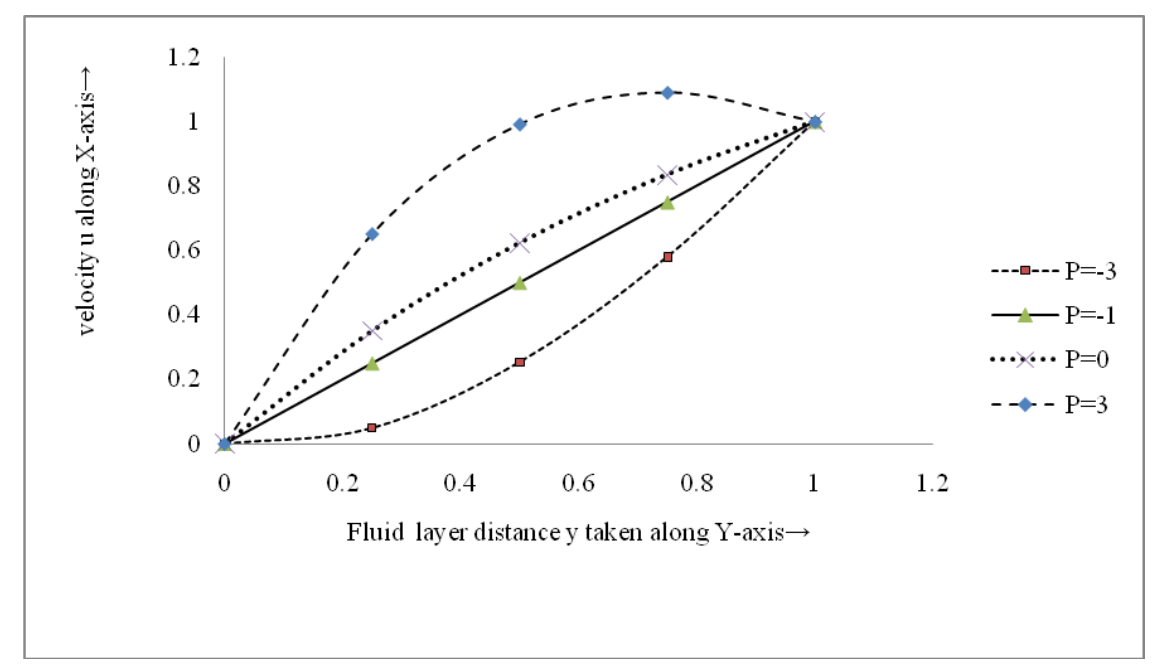

Fig. 2. Velocity profile for different values of $\mathrm{P}$ when $\mathrm{M}=0, \mu=1, \lambda=0$.

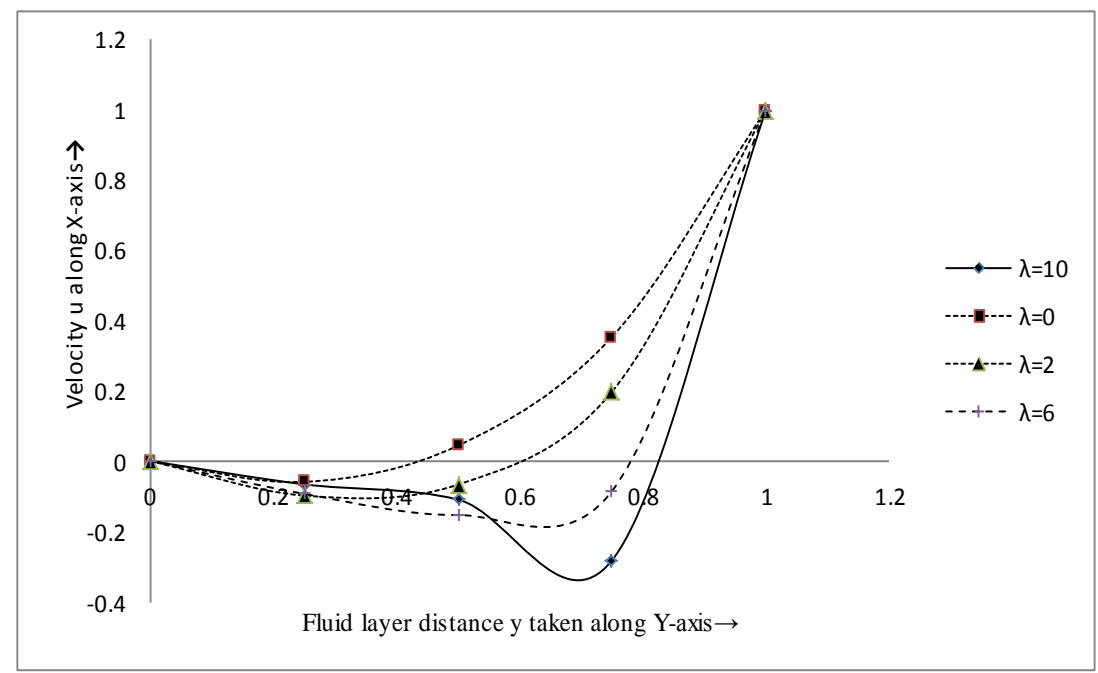

Fig. 3. Velocity profile for different values of $\lambda$ when $P=-3, \mu=1$, Ha $=3, \theta=60^{\circ}$. 
Fig. 7 reveals two facts about the relationship of magnetic inclination $\theta$ and viscosity parameter $\alpha$ on the fluid flow. It is clear that the rate of the reversed flow depth decreases with an increase of magnetic inclination $\theta$. As the rate of fluid flow is also dependent upon the viscosity of the fluid, it is observed that this rate is much more dependent upon viscosity and for a fluid of less viscosity, we get comparatively higher rate of reversed flow. The reversed flow is due to high viscosity of the fluid and its rate decreases substantially if viscosity decreases.

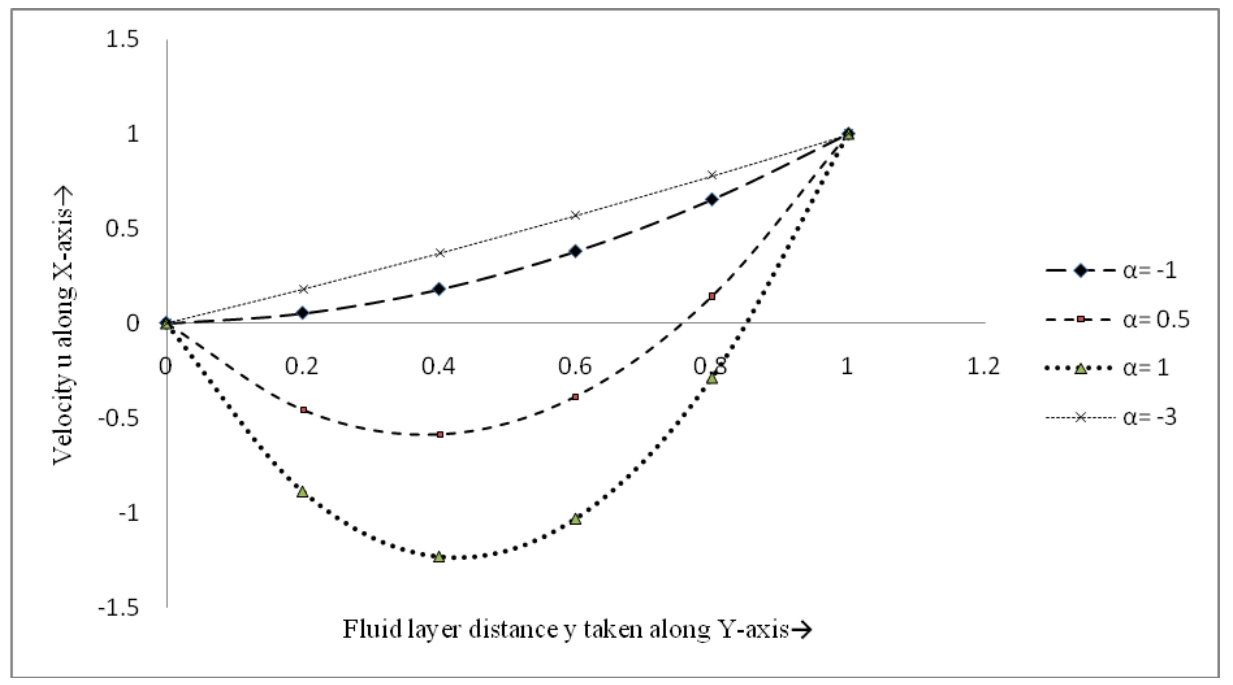

Fig. 4. Velocity profile for different values of $\alpha$ when $P=-5, M=0, \lambda=0$.

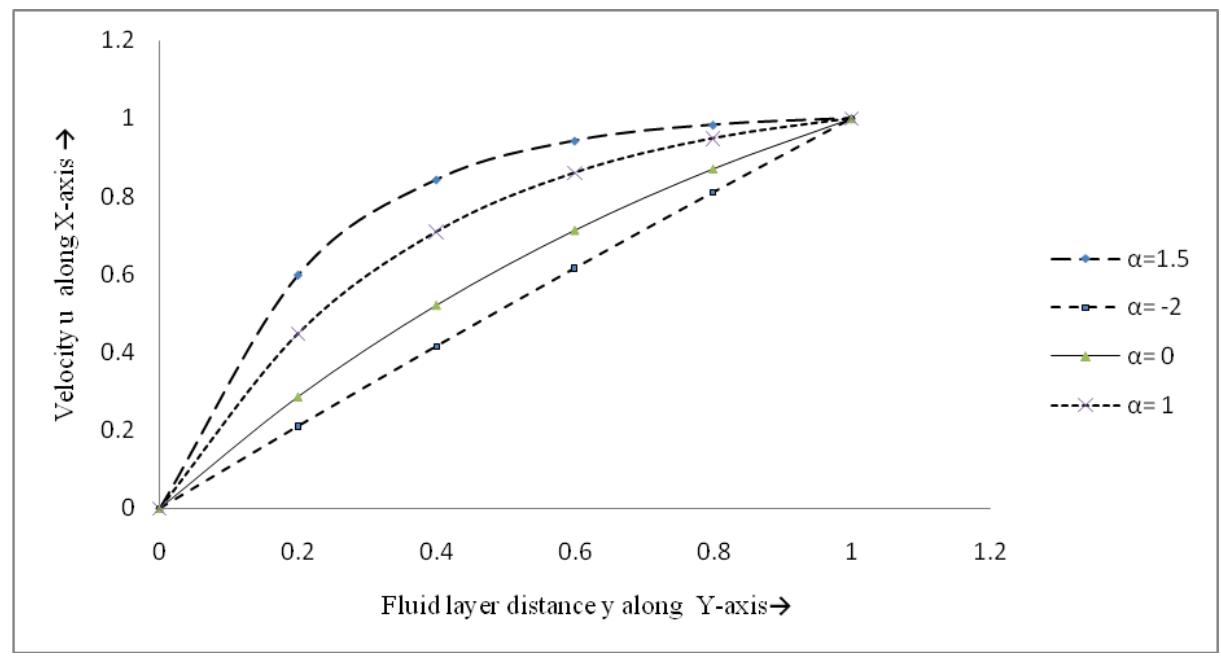

Fig. 5. Velocity profile for different values of $\alpha$ when $P=0, M=0, \lambda=0, T=1$. 
Fig. 8 indicates the influence of the viscosity parameter $\alpha$ in the temperature distribution when $P=0, M=0$, and $\lambda=0$. It is clear from the Figure that as $\alpha$ increases, the temperature decreases. Further if $\mu \rightarrow 0$, viscous dissipation occurs and in the long run the temperature profile becomes linear. Again if $\alpha$ is negative, then both the viscosity and temperature increase.

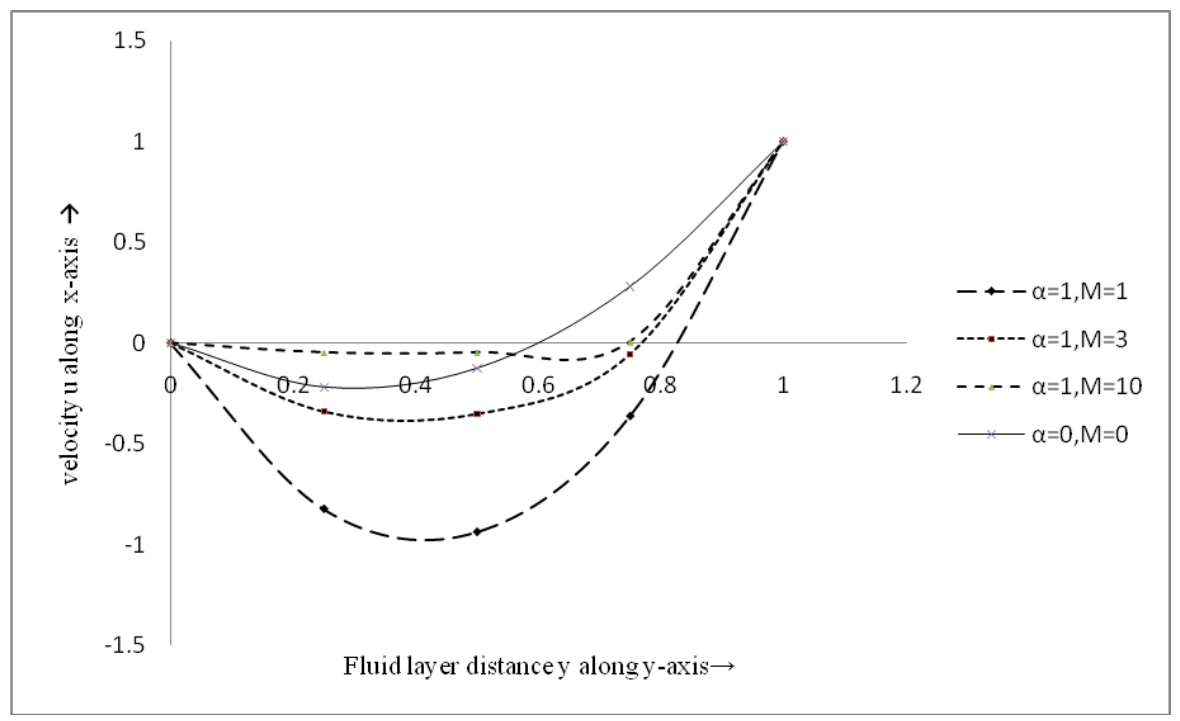

Fig. 6. Velocity profile for different values of $\alpha$ and $M$ keeping the rest parameters fixed.

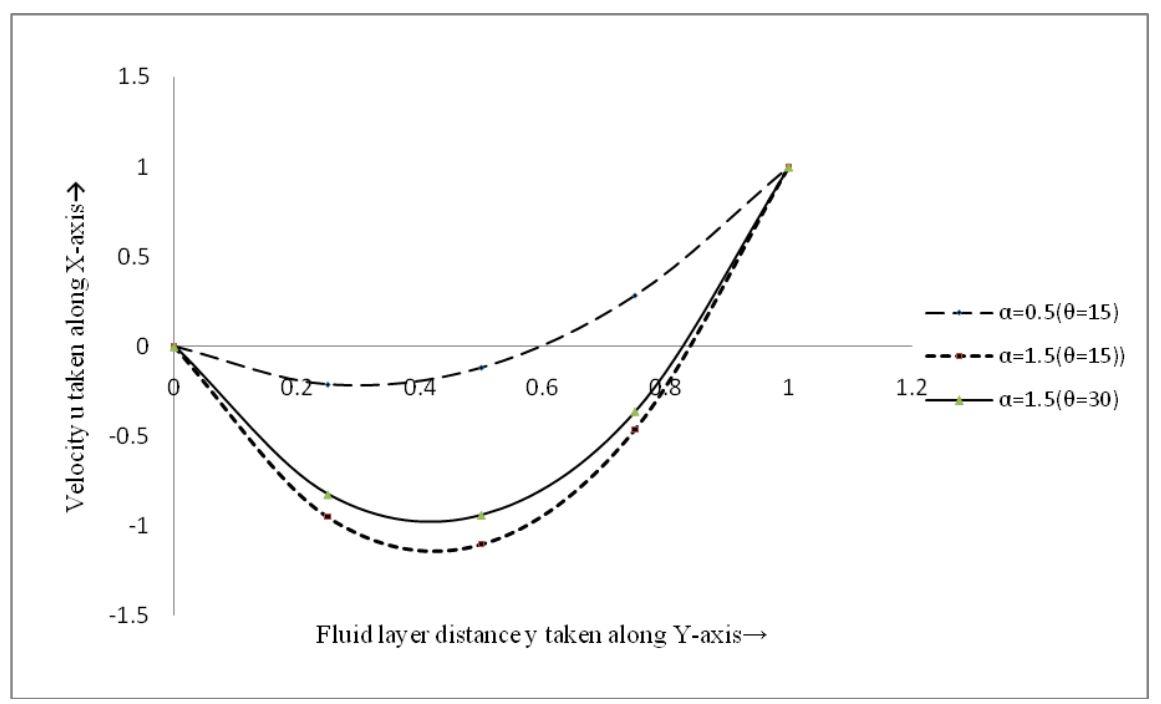

Fig. 7. Velocity profiles subject to the prescribed conditions. 


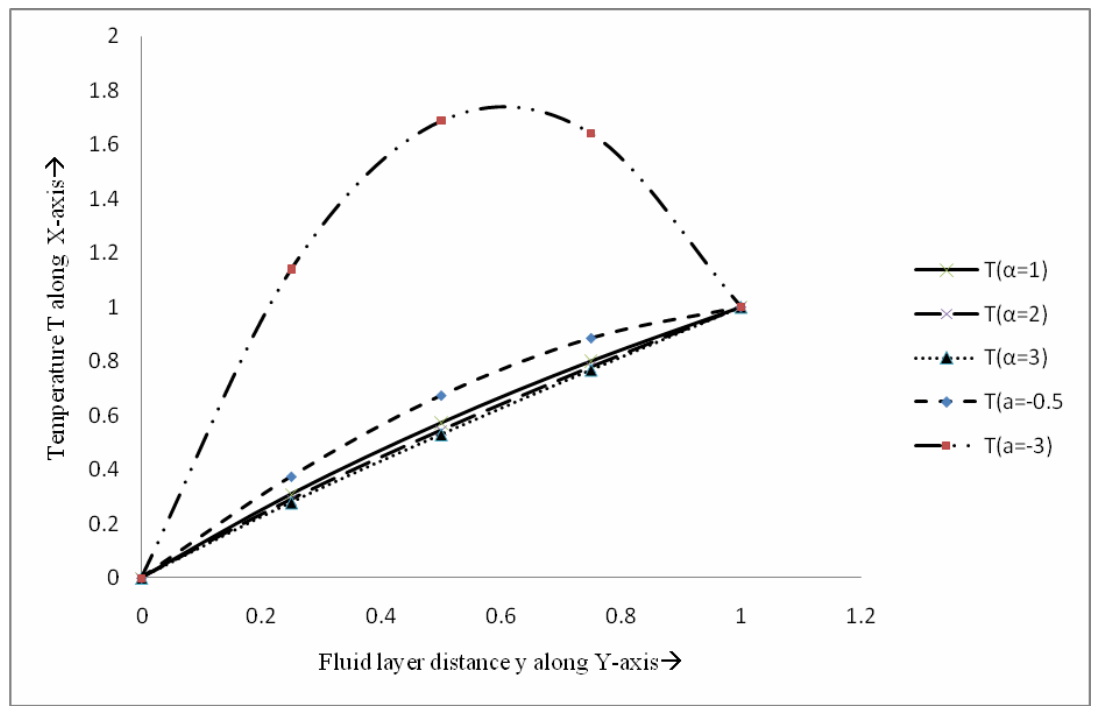

Fig. 8. Temperature profile.

\section{Conclusion}

The finite difference method is utilized to solve the momentum transfer and energy equations for the flow of an electrically conducting, viscous, incompressible fluid between two parallel infinite horizontal porous plates when the flow is considered to be laminar. The profiles for velocity and temperature are represented graphically under the influence of an external inclined uniform magnetic field, pressure gradient, inflow normal to the porous plates and temperature dependent viscosity. Comparative studies of velocity and temperature distributions in the light of magnetic inclinations have been explained for the cases of constant fluid viscosity as well as variable fluid viscosity. We observe that the parameters involved in the determination of velocity and temperature profiles play vital role in the flow. The fluid with high viscosity causes reversed flow and the applied magnetic force at different inclination controls this flow. Besides this we observe the temperature distribution in fluid layers is directly linked with its viscosity.

\section{References}

1. V. C. A. Ferraco and C. Plumpton, An Introduction to Magneto Fluid Mechanics, $2^{\text {nd }}$ edition (Oxford Clarendon press, 1966).

2. P. A. Davidson, An Introduction to MHD, $1^{\text {st }}$ edition (Cambridge University Press, UK, 2001).

3. G. W. Sutton and A. Shermann, Engineering Magnetohydrodynamics (McGraw Hill Book Company, New York, 1965).

4. J. A. Roberson and T. C Clayton, Engineering fluid mechanics (Boston, Houghton Mifflin, 1990).

5. H. Alfvén, Nature 150, 405 (1942). http://dx.doi.org/10.1038/150405d0

6. H. Stitching, Boundary Layer Theory (McGraw- Hill Book Co., New York, 1986).

7. S. I. Pai, Magnetohydrodynamics and Plasma Dynamics, (Springer verlag, Germany, 1961). 
8. R. A. Alpher, Int. J. Heat Mass Transfer 3, 108 (1960). http://dx.doi.org/10.1016/00179310(61)90073-4

9. S. D. Nigam and S. N. Singh, Quart. J. Mech. Appl. Math. 13, 85 (1960). http://dx.doi.org/10.1093/qjmam/13.1.85

10. K. Klemp, H. Herwig, and M. Selmann, in Entrance Flow in Channel with Temperature Dependent Viscosity Including Viscous Dissipation Effects - Proc. 3rd. Int. Cong. Fluid Mech. (Cairo, 1990) 3, (1990) pp. 1257-1266.

11. V. P. Carey, J. C. Mollendorf, Int. J. Heat Mass Transfer 23, 95 (1980). http://dx.doi.org/10.1016/0017-9310(80)90142-8

12. B. W. Martin, in Viscous Heating and Varying Viscosity Effects on Developing Laminar Flow in a Circular Pipe - Proc. Instn. Mech. Engrs, (1973) 187, pp. 435-445.

13. A. A. Megahed, A. L. A. Hassam, and H. S. EL-Din, in Effect of Joule and Viscous Dissipation on Temperature Distributions Through Electrically Conducting Dusty Fluid - 5th Miamic Int. Symp. on Multiphase Transport and Particulate Phenomena (Miamic Beach, Florida, USA, 1988) 3, pp. 111.

14. D. R. Kuiry and S. Bahadur, J. Math. 10, 90 (2014).

15. W. A. Manyonge, D. W. Kiema, and C. C. W. Iyaya, Int. J. Pure Appl. Maths.76, 661 (2012)

16. K. M. Joseph, S. Daniel, and G.M. Joseph, Int. J. Math. Statistics 2, 103 (2014).

17. M.Antia, Numerical Methods for Scientists and Engineers (McGraw-Hill, New Delhi, Tata, 1991).

18. R. Pyre and T. D. Taylor, Computational Methods for Fluid Flow. Springer, Germany, 1983). http://dx.doi.org/10.1007/978-3-642-85952-6 